\title{
THEOREM RELATING SPATIAL AND TEMPORAL HARMONICS FOR NUCLEAR INTERLEVEL TRANSFER DRIVEN BY COLLECTIVE ELECTRONIC OSCILLATION
}

\author{
JoHNDALE C. SOLEM \\ Theoretical Division, Los Alamos National Laboratory, Los Alamos, NM 87545, U.S.A.
}

(Received 8 October 1986; received for publication 27 November 1987)

\begin{abstract}
A crucial step in the development of gamma-ray lasers will be the discovery of an efficient mechanism for nuclear interlevel transfer. One promising mechanism uses the near-field interaction between the nucleus and laser-driven collective electronic oscillations. I prove an important theorem relating spatial and temporal harmonics: $2^{2 n}$-poles are driven only at even multiples of the laser frequency, while $2^{2 n+1}$-poles are driven only at odd multiples of the laser frequency. I comment on the theorem's relevance to a proposed interlevel-transfer experiment.
\end{abstract}

\section{INTRODUCTION}

In present gamma-ray-laser research, a good deal of attention is being given to schemes that involve a transfer of population from a long-lived isomeric state to a short-lived excited nuclear state. ${ }^{1}$ The mechanism for effecting this interlevel transfer is still a subject of speculation. Numerous mechanisms have been suggested, ${ }^{2}$ but one currently attracting interest is coupling laser-driven collective electronic oscillations ${ }^{3}$ to nuclear transitions by the near-field interaction. ${ }^{4}$ This is a scheme by which the "big antenna" of the whole atom can be made to drive the "small antenna" of the nucleus, thereby making the nucleus accessible to the intensities and quantum energies of present-day lasers.

\section{COLLECTIVE OSCILLATION}

Two important effects can be accomplished by coupling laser-driven collective electronic oscillations to the nucleus: (1) the electron motion can generate harmonics of the laser frequency and (2) the electron motion can generate higher multipolarities in the electric field. This paper deals with an important relationship between the two effects.

We need the generation of both temporal and spatial harmonics in order to drive interlevel transfer with laser radiation. Because the nuclear transfer energy is likely to be several times the laser quantum energy, the nucleus will have to couple to some multiple of the laser frequency. The laser field is almost purely dipole because the wavelength will be large compared to the atomic diameter. The transfer step is unlikely to be a dipole transition, so higher multipoles will have to be generated by the electron-cloud oscillations.

If the magnetic interaction is small, nuclear electric $2^{2 n}$-poles can be driven only at even harmonics of the laser frequency. For example, if our transition from the storage state to the upper grasing level is quadrupole, then the energy of that transition must be $2,4,6,8$, -times $h \omega$ of the driving laser. Similarly, nuclear electric $2^{2 n+1}$-poles can be driven only at odd harmonics of the laser frequency. For example, if our transfer transition is octupole, its energy must be 1, 3, 5, 7, - times $h \omega$ of the laser.

\section{Theorem}

If collective electronic oscillations are driven by radiation with wavelength long compared to atomic diameter, then the near field interaction will drive nuclear electric $2^{2 n}$-poles at even harmonics and $2^{2 n+1}$-poles at odd harmonics. 


\section{Proof}

Consider a wave $\mathbf{E}(\mathbf{r}, t)=\mathbf{E}_{0} \exp i(\mathbf{k} \cdot \mathbf{r}-\omega t)$ and an atom with electron cloud density distribution $\rho_{e}(\mathbf{r})$. If $k$ is large compared to the cloud dimensions, then $\rho_{e}(x, y, z, t)=\rho_{e}[-x, y, z, t+\pi / \omega]$, where we have chosen $\mathbf{E}(\mathbf{r}, t)=\mathbf{E}_{x} \sin \omega t$ and the oscillations of the electron cloud have been allowed to reach a steady state. Now the near-field interaction Hamiltonian between the nuclear charge and electronic charge is

$$
H=\sum_{l=1}^{\infty} \sum_{m=-l}^{m=l} \frac{4 \pi}{2 l+1} q_{l, m} Q_{l . m}+\text { inverse term }+ \text { magnetic term, }
$$

where

$$
q_{l, m}=\int \rho_{\mathrm{n}}(\mathbf{r}) Y_{l, m}^{*}(\theta, \phi) r^{l} \mathrm{~d} \Omega r^{2} \mathrm{~d} r
$$

and

$$
Q_{l, m}=\int \rho_{e}(\mathbf{r}) Y_{l, m}(\theta, \phi) r^{-1-1} \mathrm{~d} \Omega r^{2} \mathrm{~d} r
$$

$\rho_{\mathrm{n}}(\mathbf{r})$ and $\rho_{e}(\mathbf{r})$ being the nuclear and electronic charge distributions, respectively. $q_{l, m}$ is the regular nuclear multipole moment and $Q_{l, m}$ is the irregular electronic multiple moment. The inverse term, corresponding to electronic charge inside the nucleus, is small. So is the magnetic interaction term.

If $\theta$ is measured from the positive $x$ axis, then $\rho_{e}(r, \theta, \phi, t)=\rho_{e}[r, \pi-\theta, \phi, t+(\pi / \omega)]$, but the electron cloud is cylindrically symmetric around the $x$ axis, so we can also write $\rho_{e}(r, \theta, \phi, t)=\rho_{e}[r, \pi-\theta, \phi+\pi, t+(\pi / \omega)]$. The spherical harmonic parity is such that

$$
Y_{l, m}(\theta, \phi)=(-1)^{l} Y_{l, m}(\pi-\theta, \phi+\pi)
$$

so

$$
Q_{l, m}(t)=(-1)^{\prime} Q_{l, m}[t+(\pi / \omega)]
$$

The Fourier coefficients are:

$$
\begin{aligned}
& a_{p}=\frac{\omega}{\pi} \int_{-\pi / \omega}^{\pi / \omega} Q_{l, m}(t) \sin p \omega t \mathrm{~d} t \\
& b_{p}=\frac{\omega}{\pi} \int_{-\pi / \omega}^{\pi / \omega} Q_{l, m}(t) \cos p \omega t \mathrm{~d} t .
\end{aligned}
$$

Now $\sin p \omega t=(-1)^{p} \sin (p \omega t+p \pi)=(-1)^{p} \sin \{p \omega[t+(\pi / \omega)]\}$, so

$$
\int_{-\pi / \omega}^{0} Q_{l, m}(t) \sin p \omega t \mathrm{~d} t=(-1)^{l}(-1)^{p} \int_{0}^{\pi / \omega} Q_{l, m}(t) \sin p \omega t \mathrm{~d} t,
$$

and $a_{p}=0$ unless $l$ and $p$ are both odd or both even. Similarly, $\cos p \omega t=(-1)^{p} \cos \{p \omega[t+(\pi / \omega)]\}$ and $b_{p}=0$ unless $l$ and $p$ are both odd or both even.

Thus, when expanded in a Fourier series, $Q_{2 n, m}(t)$ will have nonvanishing terms only for even harmonics of $\omega$, and $Q_{2 n+1, m}(t)$ will have nonvanishing terms only for odd harmonics of $\omega$. So the nuclear electric $2^{2 n}$-poles will only couple even harmonics and the $2^{2 n+1}$-poles will only coupled odd harmonics, Q.E.D.

It is easy to show that the inverse term has the same symmetry properties. This theorem is violated only if the magnetic term is large or $\rho_{e}(\phi)$ departs substantially from $\rho_{e}(\phi+\pi)$. The extension to circular polarization is trivial. 


\section{PROPOSED INTERLEVEL TRANSFER EXPERIMENT}

Experimentalists have proposed ${ }^{5}$ to demonstrate laser-driven interlevel transfer by inducing transitions from the ground state of $U^{235}$ to the first excited state at $75 \mathrm{eV}$. Activation would be confirmed by observing the internal conversion electrons, which decay with a half-life of $26 \mathrm{~min}$. They plan to drive the transfer with a $248 \mathrm{~nm}$ laser. The 15 th harmonic is nearly resonant with the $75 \mathrm{eV}$ transition, which is an electric octupole and allowed by this theorem.

Acknowledgements - This work was supported in part by the U.S. Department of Energy, the Strategic Defense Initiative Office (IST) and managed by NRL.

\section{REFERENCES}

1. J. W. Eerkens, U.S. Pat. 3, 430,046 (1969); E. V. Baklanov and V. P. Chebotaev, Zh. Eksp. Teor. Fiz. Pism'a Red. 21, 286 (1975); P. Kamenov and T. Bonchev, C. R. Acad. Bulg. Sci. 28, 1175 (1975); L. A. Rivlin, Soviet J. Quant Electron. 8, 1412 (1977); L. A. Rivlin, Soviet J. Quant. Electron. 7, 380 (1977); B. S. Arad, S. Eliezer, and Y. Paiss, Phys. Lett. A 74, 395 (1979).

2. M. Morita, Progr. Theor. Phys. 49, 1575 (1974); C. Collins, F. Lee, D. Shemwell, and B. DePaola, J. Appl. Phys. 53, 4645 (1982); J. C. Solem, Bull. Am. Phys. Soc. 30, 1809 (1985); L. Biedenharn, K. Boyer, and J. C. Solem, Bull. Am. Phys. Soc. 30, 1822 (1985); J. C. Solem, in Advances in Laser Science, pp. 22-25, W. C. Stwalley and M. Lapp eds., AIP, New York, NY (1986); L. C. Biedenharn, K. Boyer, and J. C. Solem, ibid., pp. 50-51, W. C. Stwalley and M. Lapp eds., AIP, New York, NY (1986).

3. K. Boyer and C. K. Rhodes, Phys. Rev. Lett. 54, 1490 (1985); C. K. Rhodes, Science 30, 1345 (1985).

4. L. C. Biedenharn, G. C. Baldwin, K. Boyer, and J. C. Solem, Bull. Am. Phys. Soc. 30, 1822 (1985); L. C. Biedenharn, G. C. Baldwin, K. Boyer, and J. C. Solem, in Advances in Laser Science, pp. 52-53, W. C. Stwalley and M. Lapp eds., AIP, New York, NY (1986).

5. J. A. Bounds, P. Dyer, R. C. Haight, and S. A. Wender (private communication), Proposal to DOE/BES (1986). 